\title{
Rewiring Territories: The Future Production of Extraction Infrastructure
}

\author{
NEERAJ BHATIA \\ California College of the Arts, The Urban Works Agency
}

The disjuncture between a territorial infrastructure and local cultures is most overtly witnessed in the development of pipelines, which are large infrastructural conduits used to transport oil or gas, deployed primarily between points of extraction and refining. This article examines the typology of the pipeline and its relationship to both territorial and local systems for their eventual future productions. Specifically, it focuses on the South American "Uruguayana-Porto Alegre Pipeline", which will unify a series of separate pipelines developed over the past two decades. Spanning 3,100 kilometers across Argentina, Bolivia, Uruguay, and Brazil, and linking northern regions to the southern cone once completed, this energy ring will redistribute flows of energy, wealth, and people across the territory. As new pipelines and their associated infrastructures cross the once remote hinterland, it exposes these rural settlements and local ecologies to development pressures. This case study will use two design-research projects to investigate how the pipeline can be leveraged to reassert local cultures, economies, ecologies, and values.

\section{FUTURE PRODUCTION(S) OF LOGISTICAL ARTIFACTS}

In the context of South America, resource extraction and its associated logistical infrastructures have played a central role in organizing the territory. In many cases, the framework that these infrastructures have inscribed on the land have been appropriated by future developments and even turned into cities, such as Ciudad Ojeda and Nueva Loja. As posited by Felipe Correa, "The metrics of the oil camp have become the template for a new type of post-oil city, one that is a direct byproduct of the oil extraction process itself." ${ }^{1}$ This eventual second production is arguably contingent upon the permanence of the infrastructures and the territories they established-the logic of one system inadvertently forming the template for a future system of development. What if the design and organization of logistical infrastructures anticipated their presumable second production?
This becomes a critical question when considering that the physical artifacts, machines, and infrastructures of extraction-offshore rigs, jack pumps, pipelines, tank storage farms, refineries, transfer ports, etc.-will long outlive the resources they are exhausting. While the current lifespan of the global oil industry is estimated to be between forty and fifty-three years, geographically specific infrastructures will be used for a fraction of that time, as in the Brazilian context where this estimate drops to approximately twenty-five years. ${ }^{2}$ The most recent wave of extraction over the past ten years has been the most technologically advanced - reaching horizontally into distant geographies and vertically to enormous depths - making this final push for resources a costly venture. Yet, despite this, new infrastructures are being deployed at a faster and faster rate in service of this limited resource. Recognizing the sheer amount of infrastructure that is estimated to be constructed and the limited duration of its use, we must question how these processes and infrastructures could be organized to account for their future production(s) - a production that could comprise a more holistic set of cultural, political, ecological, and economic factors. This would position extraction infrastructures as the proactive framework for new urban organizations.

We could say that the major spatial formats of logistics consist of surfaces, containers, and conduits. ${ }^{3}$ These formats have colonized vast swatches of hinterland environments, and operate at a scale that is closely aligned to global and regional logistics. Surfaces are planes of mediation that typically function at a territorial scale as they are primarily implicated in a form of harvesting or collection. Containers are architectural shells of enclosure often sited between the formats of surfaces and conduits - for storing, refining, or distributing a particular good. The conduit as a type is tasked with interfacing between the scale of the territory and that of architecture. Conduits are used to transfer matter and energy across vast distances, cutting through local settlements, political boundaries, ecosystems, and connecting to both containers and surfaces. Conduits have a particular significance in logistical systems as they allow for the spatial separation between extraction, processing, manufacturing, and consumption. Pipelines are one of the most understudied conduits within the design disciplines, yet despite their inconspicuous nature, they are highly prevalent in our daily lives. Currently, there are five million miles of pipeline in operation around the globe, which, if connected 


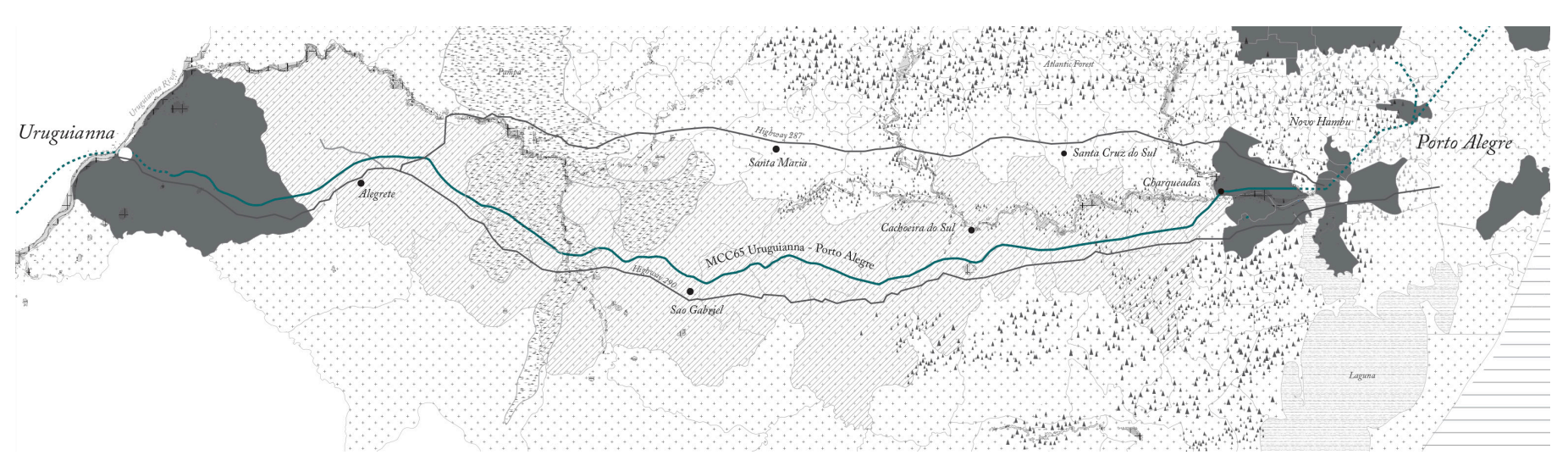

Figure 1: MCC65 Pipeline moving through the Brazilian hinterland, its local settIments and different ecological biomes (Drawing by: Cesar Lopez/ The Open Workshop.

end-to-end, would wrap the equator two hundred times. ${ }^{4}$ How can we leverage this territorial infrastructure to foster new relationships between resources, cultures, and economies once oil is depleted?

\section{DISSOCIATED TECHNOLOGY}

Energy pipelines are large infrastructural conduits used to transport oil or gas, deployed primarily between points of extraction and refining. Pipelines are desirable because they are able to move energy in a more efficient, reliable, and safer manner than tanker trucks or rail tanker cars. ${ }^{5}$ Beyond safety, the pipeline was a critical invention in making energy an abstract commodity by separating sites of extraction from those of consumption; operating almost invisibly with little human interaction. ${ }^{6}$ This enabled the environmental costs of oil extraction to be highly removed - allowing those that benefited from cheap energy to not have to confront the environmental consequences of its extraction. The abstraction fostered by spatial detachment has characterized the pipeline as a dissociated technology. ${ }^{7}$

As a dissociated technology, the physical tension between local conditions and the endless extrusion of the artifact can be witnessed both at the scale of the region as well as the detail. Regionally, pipelines are but one component in the larger linear infrastructure of the easement, a zone approximately seventy-five meters in width that is cleared to neutralize and tame the varied conditions that are to be navigated. The easement becomes the primary corridor for access, communication, maintenance, surveillance, and construction. Moreover, compared to the pipeline itself, the easement creates perhaps the most permanent condition of all - these zones are often deemed their own political-economic jurisdiction (free-trade zone), a designation that attracts other infrastructures and activities that solidify particular characteristics within this vector. Accordingly, the rules governing these thickened lines are established through global concerns and rarely bring benefit to local communities or ecologies. At the scale of the detail, it is the technical engineering and properties of fluid dynamics that determine the internal specifications of the pipe - including the diameter, thickness, material selection, fittings, etc. While the outside of the pipeline inevitably interfaces with the external environment, these conditions are typically viewed as secondary to the internal specifications and thereby addressed through mitigation techniques. What if the physical environment and its inhabitants played a larger role in determining the location, trajectory, and design of pipelines and their associated infrastructures? This would bring into consideration the settlements and ecologies that will ultimately inherit these infrastructures and potentially utilize them for differing means. This could reframe these infrastructures as associative technologies - moving resources to connect people and environments while also providing legibility to logistical processes and thereby reducing their abstract logic. Through clarifying once abstract territorial processes, one is made cognizant of their affects (both positive and negative). Our contention here is that the capacity for someone to physically 'read' the components and relationships within a logistical system, is critical to an awareness of one's position within this territorial arrangement. This understanding has deeper effects on how one acts, is accommodated, and ultimately, empowered within urban space-even when this space is organized at the scale of the territory.

The interdependencies between sites of oil and gas extraction, refining, and consumption have fostered a network of territorial conduits, which move through the South American hinterland, including its communities and ecologies. Attempting to shed light on the above questions through design, we focused our case study on the Uruguayana-Porto Alegre Pipeline (also termed MCC65), which will unify a series of separate energy pipelines developed over the past two decades into an 'energy ring'. Spanning over 3,100 kilometers and running through Brazil, Argentina, Bolivia and Uruguay, this circuit will link northern regions to the southern cone once completed. The last remaining segment, $\mathrm{MCC} 65$, will transverse 570 kilometers across Southern Brazil and terminate in Uruguay. This journey crosses vastly different conditions from the developed and industrialized coast to the agrarian hinterland. The following two case studies examine how industry, ecologies, and local cultures and economies can take control of these territorial artifacts and machines. 


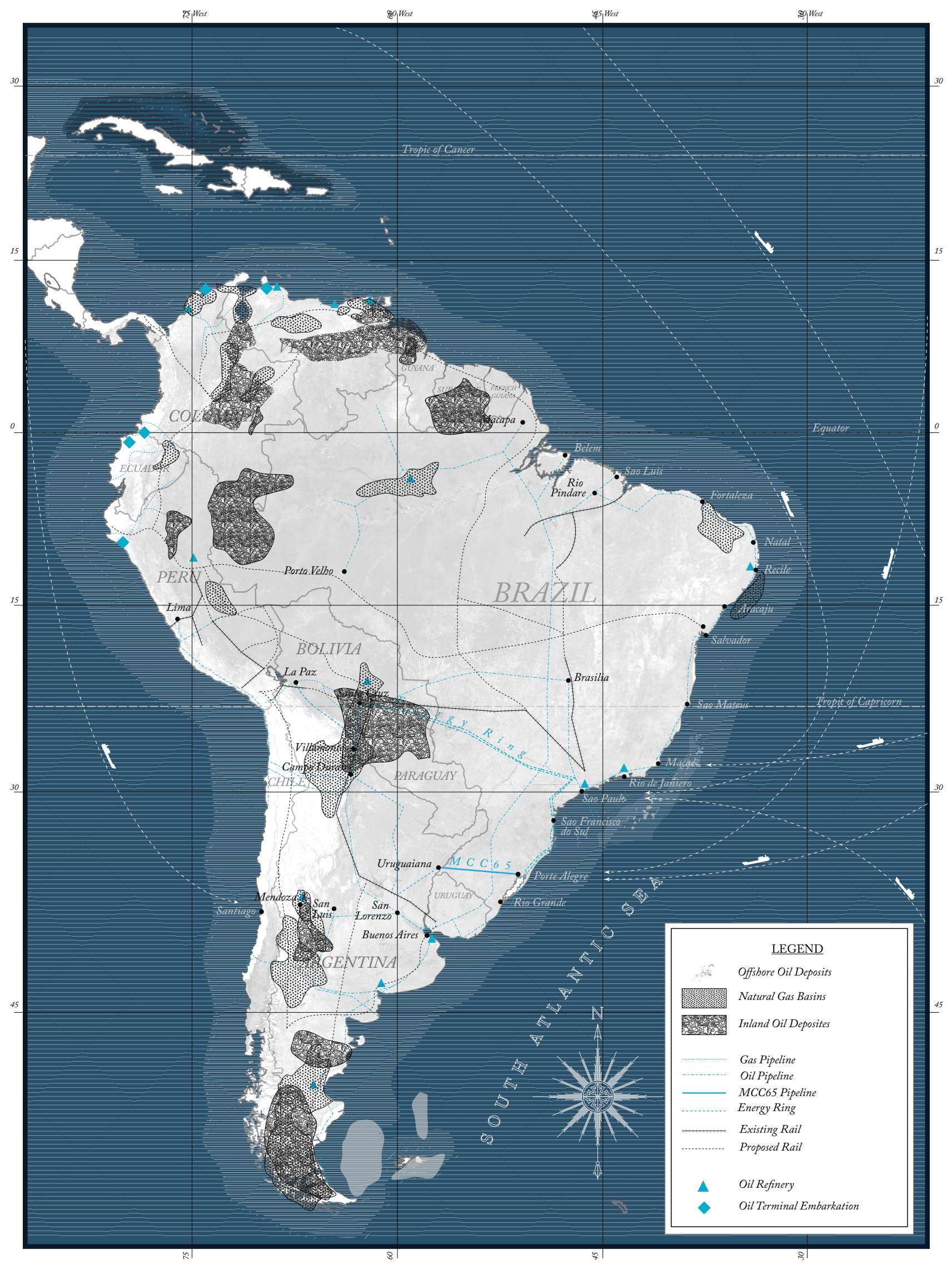

Figure 2: Sites of oil and gas extraction as well as pipeline conduits in South America (Drawing by: The Open Workshop / Cesar Lopez) 


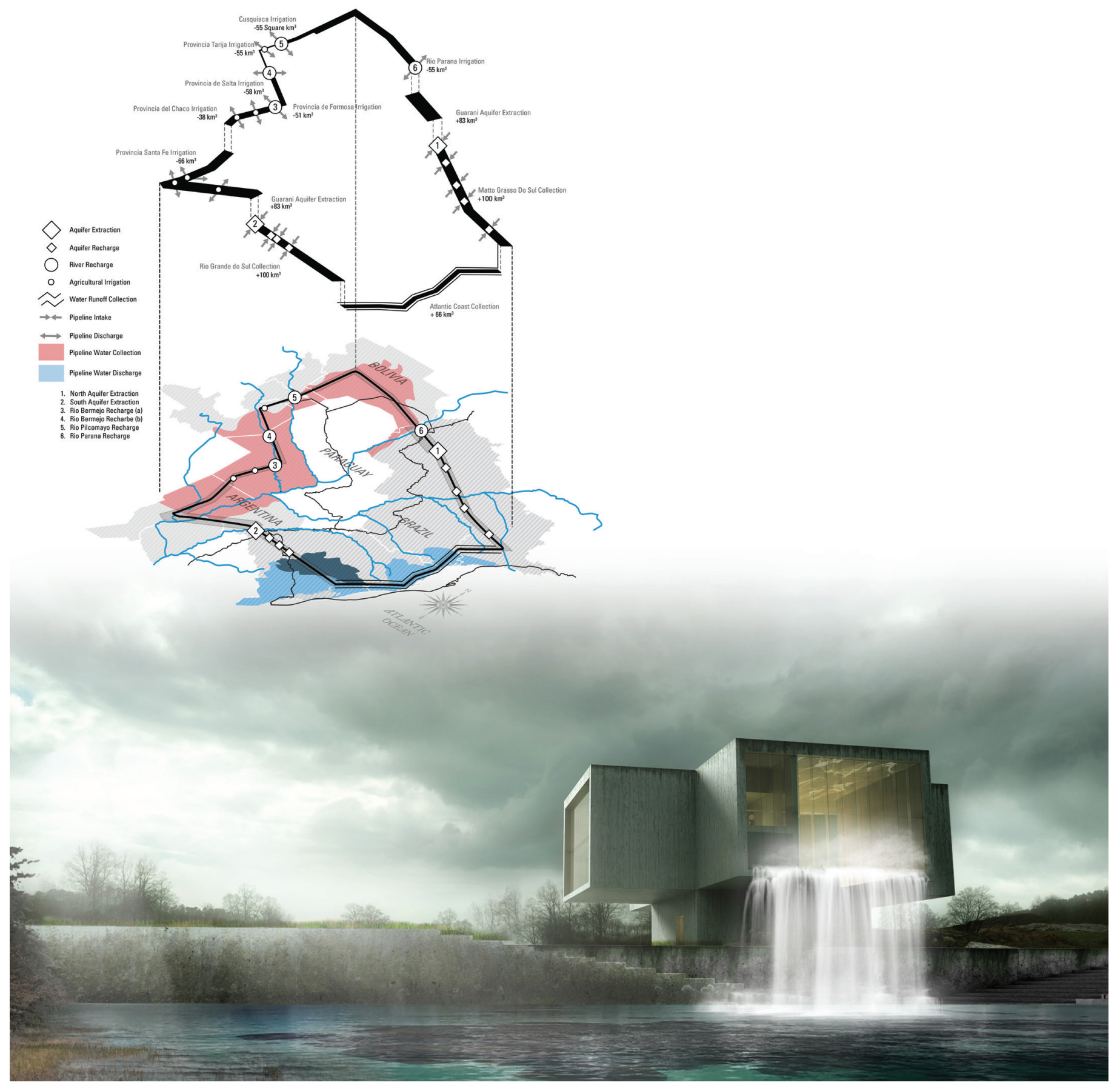

Figure 3: Inputs and outputs of the energy ring (top left), view of discharge node into the remediated mine/ aquifer recharge point. (Blake Stevenson, Lujac Desautel)

\section{REWIRING HYDROLOGY}

Hydroscapes is a proposal that leverages the territorial connectivity of the pipeline and its easement for a second production of sustainable water management, which benefits new subjects while making legible key components within the logistical process. The lifespan of fossil fuels within this geographic context coincides with a tipping point in water availability and food scarcity. ${ }^{8}$ Climate models anticipate that the isolated inland region known as the Gran Chaco - spanning from northwestern Argentina through western Paraguay and Brazil - is in a state of increasing desertification that results in reduced agricultural productivity. The Gran Chaco has been in a rapid state of decline since its very settlement in the late 19th century by colonizers exploiting the abundant hardwoods of the region while securing additional land for cattle grazing. ${ }^{9}$ Nearly three million hectares of forest have been lost from the Chaco between 1990 and 2011, largely due to the expansion of irresponsible farming techniques that have precipitated soil erosion and groundwater salinification. ${ }^{10}$ Moreover, the lack of infrastructural connections into this region has limited the economic output for the locally deprived inhabitants of the Gran Chao. As a consequence, the 


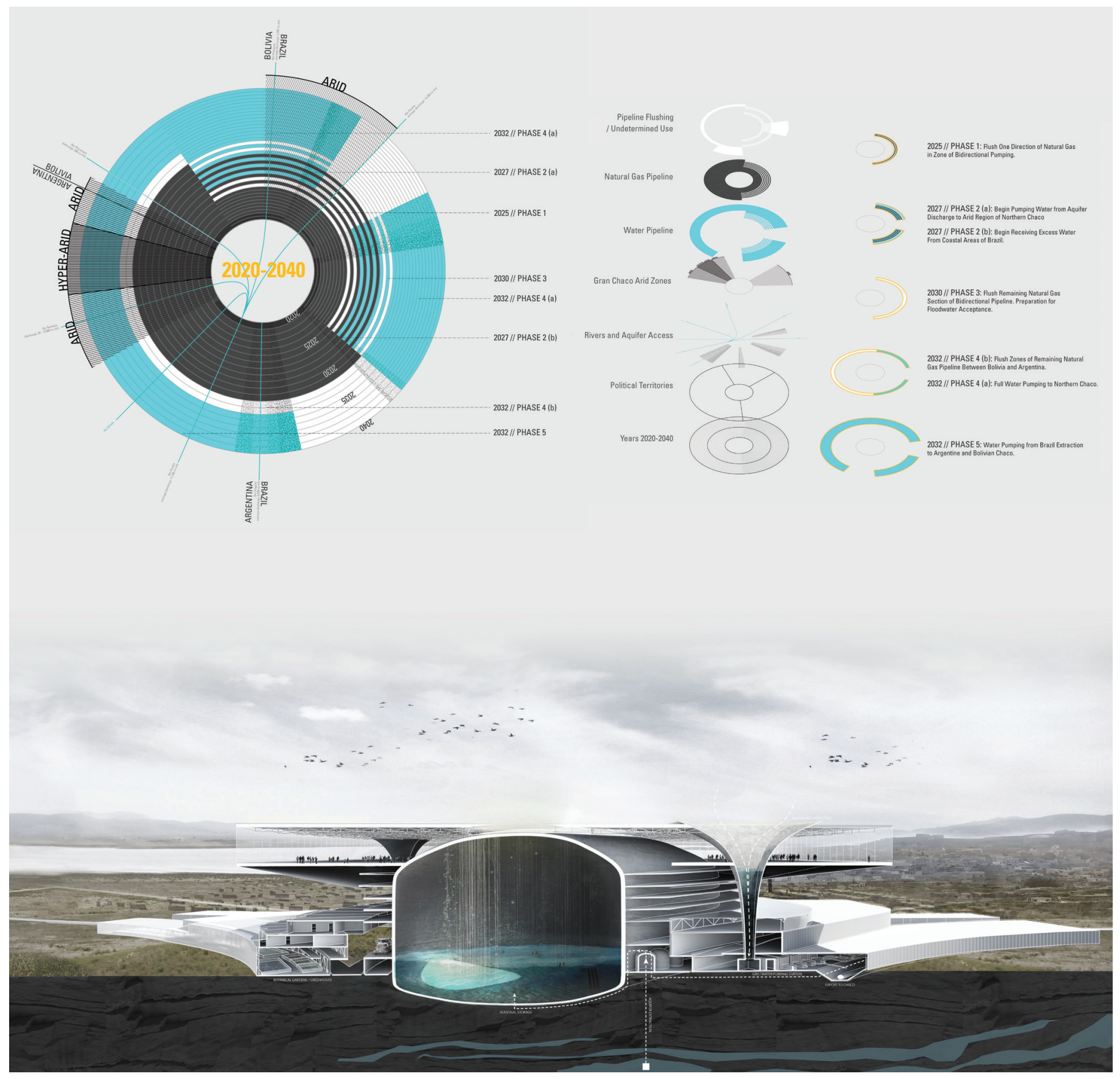

Figure 4: Phasing of the pipeline from energy to water (top), view of extraction point / food logistics center (Blake Stevenson)

already isolated Gran Chaco is suffering from a lack of agricultural productivity, making it fragile to cope with projections of drought. Conversely, along the Eastern Coast of Brazil and its string of coastal cities, climate change models are anticipating an increase in precipitation and unprecedented flooding as the watershed drains into the ocean. ${ }^{11}$ The proposed pipeline not only traverses these complex ecologies related to both water scarcity and surplus, it provides new infrastructural access and connection into the hinterland. Re-operationalizing the pipeline by transitioning from fossil fuels to water transport and management has the ability to reconcile territorial water imbalances while leveraging this global infrastructure to benefit some of the most deprived citizens and damaged landscapes in South America. ${ }^{12}$

Spanning between and below these diverse regions is the world's second largest groundwater source - the Guarani Aquifer. This aquifer contains nearly ten times more water than the Ogallala Aquifer in the American Midwest, which has been a critical resource to activate the American agricultural economy. While the Ogallala has a low recharge rate and is swiftly diminishing, the Guarani has a heavy recharge rate and can maintain a volume of water to supply fresh drinking water to the present population for up to 1,600 years. ${ }^{13}$ The potential of the Guarani Aquifer lies in its ability to saturate the arid soils of the Gran Chaco, yet historically this region has lacked appropriate management to ensure it 


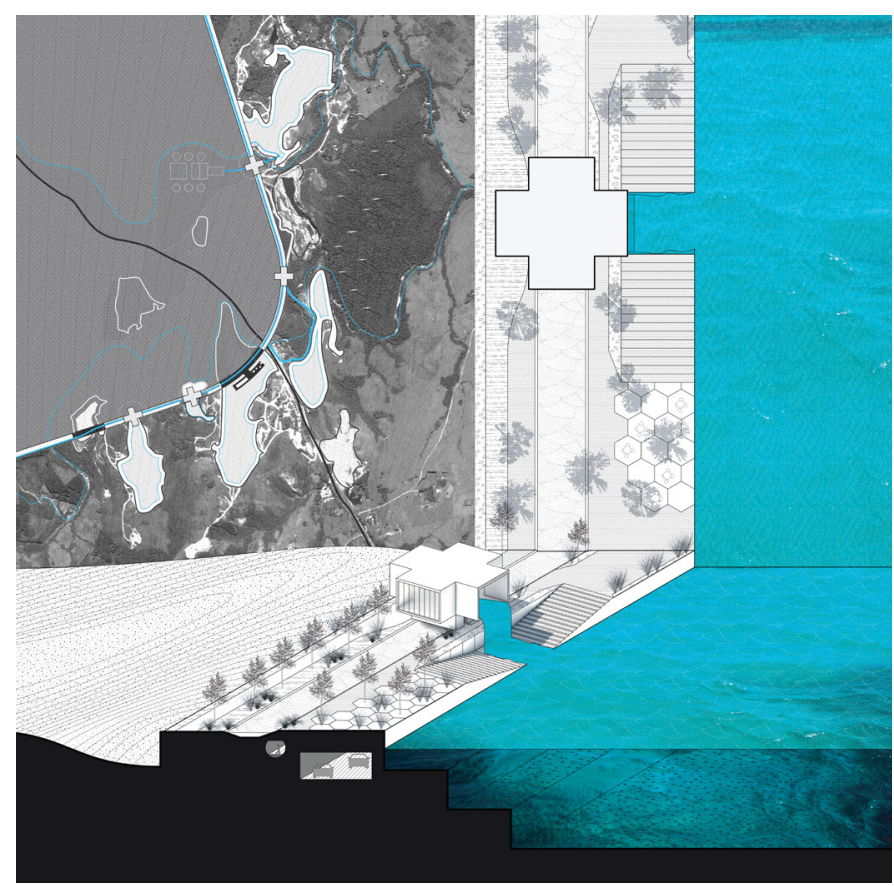

Figure 5: Cacapava do Sul waterbanking sites from former mine quarries. (Lujac Desautel)

avoids over extraction and salinification. Hydroscapes utilizes the pipeline in combination with the aquifer to establish a sustainable water management system - balancing extraction and recharge. The aim of hydroscaping the larger territory is not to simply reverse climatic trends, but rather to empower local economies in the hinterland that might benefit from the pipeline. Additionally, the project seeks to reduce the abstraction of these infrastructures through an architecture that celebrates an increasingly scarce and volatile resource, creating legibility at the scale of the individual and territory they belong to. The proposal consists of critical nodes linked to specific geographies of the energy ring - collection and remediation along the Atlantic coast through the reoperationalizing of pipelines and easements; water banking and aquifer recharge on the coastal interior in former mine quarries, water extraction and food logistics along the western edge of the aquifer, and finally surface recharge sites that interface with natural rivers.

The primary region for capturing water would be the eastern portions of the energy ring that run along the Brazilian coast and cross the Atlantic watershed of Southern Brazil. By redeveloping the pipeline's easement as a phytoremediation wetland and detention basin, this vast linear territory would capture, cleanse, and hold the increased predicted water runoff while mitigating flood threats to coastal cities. Water would then be moved by culverts into the main pipeline and pumped westwards to regions of water scarcity. This collected water would primarily be utilized to ballast aquifer extraction, recharging the groundwater with remediated runoff. These sections of the pipeline and easement on the eastern edge of the energy ring could transition resources as soon as 2030, after the pipeline is absolved of fossil fuels.

Water banking would take place on the coastal interior in areas above the aquifer, and employ former mining sites as recharge points. Passing through Brazil's hinterland in Rio Grande do Sul, the

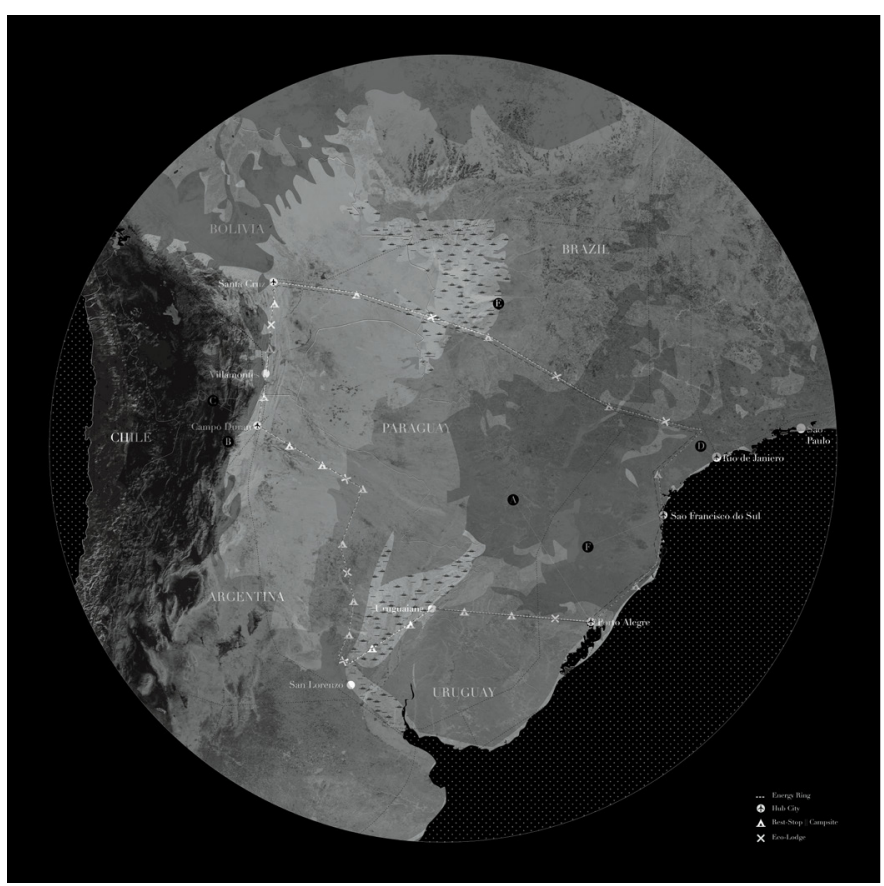

Figure 6: Eco-tourism trail network rooted with the energy easement (Enrique Justicia, Jill Chao)

MCC65 pipeline encounters a series of settlements that depend on the extraction of coal, copper, gold, and stone. Massive pits in the earth produced by these processes descend toward the Guarani Aquifer beneath. These resources, like fossil fuels, are quickly being exhausted and threatening local economies unless they can adapt and diversify. For instance, between 2020 and 2025, the mines of Cacapava do Sul that sit along the pipeline's path will be obsolete and the lands of its settlement, currently with a population of over 30,000 people, contaminated. By remediating these mining pits, these sites could be leveraged for water banking and aquifer recharge. This would shift the cultural and economic identity of the settlement from one dependent on global resource extraction to one rooted in local ecotourism and recreation, which is already an emerging economy in the region. Introducing cultural and ecological programs to these formerly hidden sites invites local subjects to engage with the territorial systems that surround them. Further, it would create moments of territorial visibility using architecture at the key point of water transfer between the pipeline and aquifer, offering a legibility to the past and present processes running through the site. Both of these tactics reduce the abstraction of these infrastructures and allow local subjects to find opportunity by locating themselves within the logistics of the territory.

Water banking would take place on the coastal interior in areas above the aquifer, and employ former mining sites as recharge points. Passing through Brazil's hinterland in Rio Grande do Sul, the MCC65 pipeline encounters a series of settlements that depend on the extraction of coal, copper, gold, and stone. Massive pits in the earth produced by these processes descend toward the Guarani Aquifer beneath. These resources, like fossil fuels, are quickly being exhausted and threatening local economies unless they can adapt 


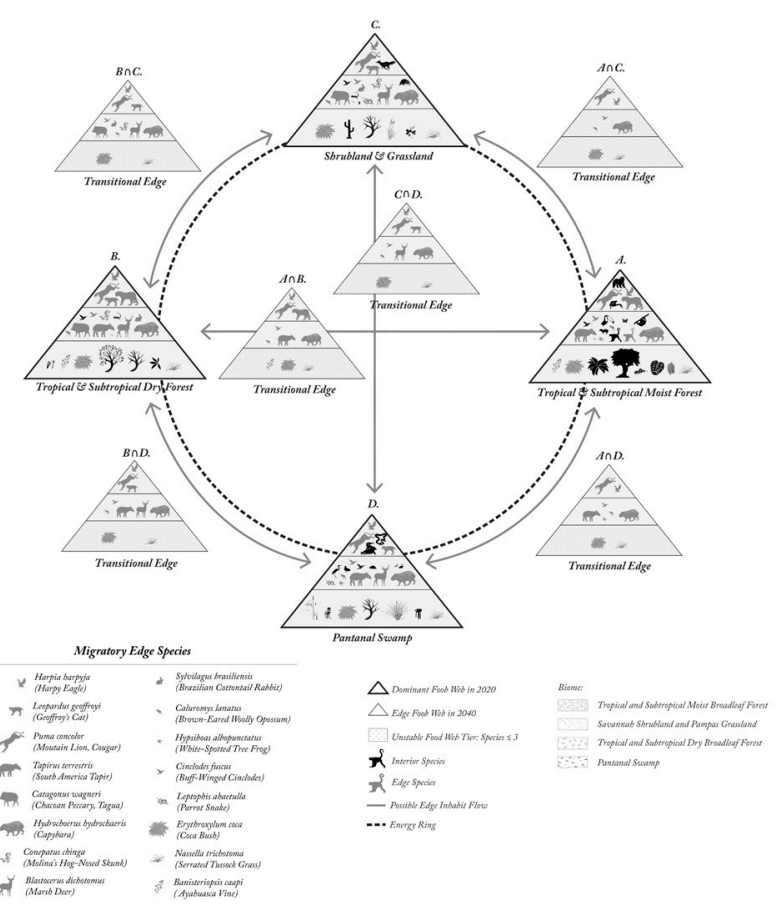

Figure 7: New food webs and interactions created by interior / edge species re-negotiation from the pipeline and easement (Enrique Justicia, Jill Chao)

and diversify. For instance, between 2020 and 2025, the mines of Cacapava do Sul that sit along the pipeline's path will be obsolete and the lands of its settlement, currently with a population of over 30,000 people, contaminated. By remediating these mining pits, these sites could be leveraged for water banking and aquifer recharge. This would shift the cultural and economic identity of the settlement from one dependent on global resource extraction to one rooted in local ecotourism and recreation, which is already an emerging economy in the region. Introducing cultural and ecological programs to these formerly hidden sites invites local subjects to engage with the territorial systems that surround them. Further, it would create moments of territorial visibility using architecture at the key point of water transfer between the pipeline and aquifer, offering a legibility to the past and present processes running through the site. Both of these tactics reduce the abstraction of these infrastructures and allow local subjects to find opportunity by locating themselves within the logistics of the territory.

Moving deeper into the hinterland by the Western edges of the aquifer, a series of water extraction sites are located, which tap into the aquifer and transport its water further inland to the Gran Chaco. One such site is in the city of Uruguayana, which runs along the BrazilArgentina border. Uruguayana is one of the most infrastructurally connected cities in the Brazilian hinterland; a series of highways and rail lines converge in the city to feed the largest dry dock in South America and third largest in the world. Acting as a critical node to gather and move agricultural products to the eastern coastal cities, the logistical hub and transfer station has a direct relationship to the suffering agricultural regions that benefit from extracting groundwater at this site. The compressor station for energy transport is easily retrofitted into a pumping station for the distribution of water to the east. This extraction point would also be designed to house the region's largest food distribution center and public marketplace. Overflowing seasonally with the consolidated products from the Gran Chaco such as cotton, sorghum, sugarcane, soy, maize, beans, and rice, this exchange point will be visible at great distances from the infrastructures that gather at this node.

Once extracted from the aquifer, water exits the MCC65 section of the pipeline and enters the larger energy ring, moving towards the Gran Chaco and eventually discharged into four natural rivers - the Rio Bermejo, Rio Pilcomayo, Rio Paraguay, and Rio Parana. Each of these rivers acts as a conduit for surface recharge - irrigating the desertifying soils of the six provinces in Argentina, Brazil and Paraguay lying within the Chaco territory. The point of discharge - the intersection between natural hydrological systems and the pipeline that reinvigorates them - is punctuated in the proposal with architectural form.

\section{REWIRING ECOLOGY}

The insertion of oil and gas pipelines across a vast landscape introduces a range of ecological issues. This is primarily caused by the large width of the easement, which is clear-cut across the territory to enable access, maintenance, and surveillance. Issues from pipeline construction alone can instigate the loss of native vegetation species diversity, invasive species, loss and fragmentation of habitats, alteration of drainage patterns, as well as new human access to previously undisturbed areas. ${ }^{14}$ One of the most pronounced concerns is the fragmentation of biomes. Fragmentation refers to "a reduction of the initial area covered by a natural habitat in a landscape, and an alteration of the habitat structure, where the remaining habitat is divided into smaller and more insulated portions ${ }^{15}$ As pipelines and their associated easement cut through a biome, they destroy habitats for interior species, and create more areas for edge species. ${ }^{16}$ Additionally, this phenomenon disrupts the typical food web, introduces new predatory species, and transforms migratory patterns of species. Within this third space of the easement, new migration paths are formed, which create unique hybrid food webs between species that are able to move more fluidly between biomes. ${ }^{17}$ Migratory Ecologics is a proposal that asks how this unique ecosystem within the third space could be made more robust while strengthening the interior of the biomes.

One of the most effective stewards of the hinterland landscape in South America is through eco-tourism. Studies have shown this to be particularly true when the industry is small-scale and locally operated and owned. ${ }^{18}$ Descending into a particular location, and using this point as a base for daily hiking typically defines eco-tourism's experiential structure. Instead of this model, Migratory Ecologics examines how the easement can be designed to allow for the co-existence of migratory species as well as eco-tourists. In this manner, eco-tourism is set up as a series of trails that take extraordinary cross-sections through the South American landscape. Variable hierarchies of pumping stations are coupled with different rest stops for hikers. The economic gains through introducing eco-tourism through this third space are used to manage, conserve, and 


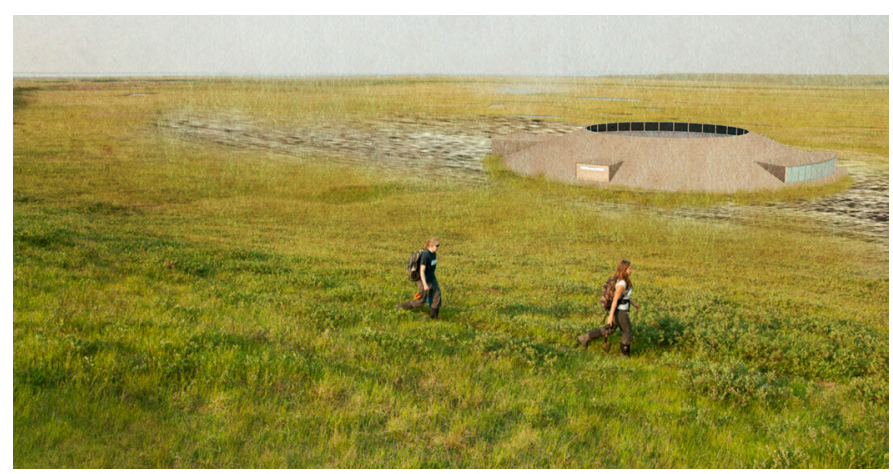

Figure 8: Vlew of the Eco-hut research center and rest stop (Enrique Justicia)

protect the surrounding landscape. Within this third space of the pipeline's easement the surreal confluence of migrating species, tourists, local operators and energy find a surreal resonance.

Different nodes along this super-ring of pipelines are developed into areas or rest, research, or ecologic choreography. Eco-Junction is sited where the introduction of the pipeline fragmented the Atlantic Forest biome into a series of islands. Strategically located as an inter-habitat bridge for interior species that typically do not migrate, the project examines how to create artificial interiors of biomes that are able to cross the pipeline. This transverse corridor connection to interior habitat patches have been studied and modeled in other parts of the globe such as California. ${ }^{19}$

Conceived of as a rest-stop/research station along the pipeline's route, the Eco-Hut proposal plugs-in to the energy ring as a secondary node. Making reference to tribal building traditions in the area, the structure embeds itself with its surroundings, concealing its presence from the animals as a way minimizing its physical and visual impact. The allocation of program forces interaction between the researcher and the tourist by organizing the guest rooms and research labs around a collective space that is supported by smaller common areas. Through these moments of engagement the tourist acquires a higher understanding of the local flora and fauna and the impact of pipeline infrastructures on natural ecosystems. Ultimately, Migratory Ecologics is a study on how to leverage new mutant ecologies produced by large-scaled infrastructures as a way to protect and conserve untouched landscapes as well as promote new forms of human interaction, engagement, and learning.

\section{ASSOCIATIVE TECHNOLOGY}

Phasing the South American energy ring into an aqueduct or hiking trail does more than simply combat the negative effects of climate change-it challenges the existing methods of land and water management, suggesting instead a system of adaptation through infrastructural reuse. This exploration into retrofitting fossil fuel infrastructure attempts to rewire the relationship between conduits, economies, local communities, and resources. Establishing a second production that finds opportunity in territorial imbalances, it bestows the benefits of these differences to strengthen local communities and ecologies. The vast scale and abstract nature of these relationships is clarified and made legible through discrete moments of architectural form. The transition from the dissociated technology of globalized logistics to an associative and empowering technology that links and makes visible local inhabitant's relationship to water as well as each other, is ultimately a socio-political question of design.

Design Projects were developed in an Advanced Studio run by Neeraj Bhatia at the California College of the Arts in 2014. Project Designers: Blake Stevenson \& Lujac Desautel (Hydroscapes), Jill Chao \& Enrique Justicia (Migratory Ecologics

\section{ENDNOTES}

1. Felipe Correa, "Afterlife Strategies: The Other Post-Oil City", Volume, Nr. 29 (2001): 130.

2. OLydia Smith, "World Energy Day 2014: How Much Oil is Left and How Long Will It Last?", International Business Times (October 22, 2014), http://www.ibtimes.co.uk/ world-energy-day-2014-how-much-oil-left-how-long-will-it-last-1471200

3. Neeraj Bhatia et al., "Formatting Contingency" in: Pamphlet Architecture 30: Coupling (New York, Princeton Architectural Press, 2011), 8.

4. Thomas O. Miesner and William L. Leffler, Oil and Gas Pipelines, (Tulsa: PenWell Books, 2006), 213.

5. Morgan Downey, Oil 101 (New York: Wooden Table Press, 2009), 257.

6. Christopher F. Jone, "Pipelines and Power", Routes of Power (Cambridge: Harvard University Press, 2014), 143.

7. Ibid., 143, 144.

8. Ximena Loza, "Paraguay, The People's Tenacity Revives EI Chaco", World Food Programme, https://www.wfp.org/stories/ paraguay-people\%E2\%80\%99s-tenacity-revives-el-chaco

9. Dr Fernando Riveros, "The Gran Chaco", Food and Agricultural Organization of the United Nations, http://www.fao.org/ag/AGP/agpc/doc/Bulletin/Granchaco.htm

10. Walcott, J., J. Thorley, V. Kapos, L. Miles, S. Woroniecki and R. Blaney (2015). Mapping multiple benefits of REDD+ in Paraguay: using spatial information to support land-use planning. Cambridge, UK: UNEP-WCMC.

11. Centro de Estudos de Geologia Costeira e Oceânica - CECO, UFRGS, toldo@ufrgs.br

12. A precedent of this transition can be witnessed in parts of the TransArabian Pipeline. See: Aaron Wolf, "Water for Peace in the Jordan River Watershed," Natural Resources Journal 33 (Summer 1993): 797-839 and Neeraj Bhatia, "Harvesting Urbanism through Territorial Logistics", The Petropolis of Tomorrow (New York: Actar Publishers, 2013), 284-285.

13. "Guarani Aquifer", World Heritage Encyclopedia, http://cn.worldheritage.org/ articles/Guarani_Aquifer

14. Marilyn Neville (Graminae Consulting), Best Management Practices for Pipeline Construction in Native Prairie Environments: A Guide for Minimizing the Impact of Pipeline Construction on the Native Prairie Ecosystem (Edmonton: Alberta Environment \& Alberta Sustainable Resource Development, 2002), 23-24.

15. Linnéa Johansson, Conservation Aspects for a Fragment of Araucaria Moist Forest in Southern Brazil: Regarding Species Composition and Diversity of a Small Fragment of Araucaria Moist Forest embedded in a matrix of Pampas Grassland, (Bachelors of Biology Thesis) (Halmstad: Halmstad University, 2013), 4

16. Ibid, 6. See also: Maíra Taquiguthi Ribeiro, Flavio Nunes Ramos, Flavio Antonio Maës Dos Santos, Tree structure and richness in an Atlantic Forest fragment: distance from anthropogenic and natural edges (Sociedade de Investigações Florestais Vicosa, Brasil, Revista Árvore, vol. 33, Issue. 6, November-December 2009), 1124.

17. Ibid, 6-7.

18. Hearne, R., Santos, A., 2005. Tourists and locals preferences toward ecotourism development in the Maya Biosphere Reserve, Guatemala. Environment, Development and Sustainability 7 (3), 303-318 and Hinch, T., 1998. Ecotourists and indigenous hosts: diverging views on their relationship with nature. Current Issues in Tourism 1 (1), 120-124.

19. Spencer, W.D., P. Beier, K. Penrod, K. Winters, C. Paulman, H. Rustigian-Romsos, J. Strittholt, M. Parisi, and A. Pettler, California Essential Habitat Connectivity Project: A Strategy for Conserving a Connected California. (Sacramento: California Department of Transportation, California Department of Fish and Game, and Federal Highways Administration, 2010), 125. 